\title{
Evaluation of MALDI-TOF MS in the microbiology laboratory
}

\section{Avaliação da técnica de MALDI-TOF MS no laboratório de microbiologia}

\author{
Anderson Fernandes Santos ${ }^{1}$; Rodrigo Cayố2; Lygia Schandert ${ }^{3}$; Ana Cristina Gales ${ }^{4}$
}

\begin{abstract}
Rapid identification of microorganisms by the clinical microbiology laboratory is of crucial importance for optimal patients' management and treatment. In general, bacterial identification by conventional methods requires 18-24 hours for colony isolation and at least 24 additional hours for species identification. New technologies in microbiology have focused on the rapid diagnosis of bloodstream infections, since they are associated with high morbidity and mortality rates.
\end{abstract}

Key words: MALDI-TOF MS; mass spectrometry; blood stream infection; bacterial infections; rapid diagnostic.

\section{INTRODUCTION}

Clinical microbiology has developed continuously and there has been a constant search for new techniques and diagnostic methods with the aim to optimize the identification of pathogens. The rapid and accurate identification of infectious agents is of crucial importance in the clinical practice, mainly in serious infections such as blood stream infection (BSI), which may progress to sepsis/septic shock, with high morbidity and mortality rates. Projections have demonstrated that BSI incidence may rise by at least $10 \%$ annually in the next years, causing a major social and economic impact on the health care system ${ }^{(1,3,6-8,15)}$.

The accurate microorganism identification and the early introduction of an appropriate antimicrobial therapy are related to better clinical outcome of septic patients. According to Kumar et al. ${ }^{(19)}$, the death risk increases by $7 \%$ after each hour between the beginning of the septic shock and the adjustment of antimicrobial therapy ${ }^{(19)}$. Furthermore, other studies have substantiated the reduction in costs with the use of an appropriate antibiotic therapy ${ }^{(9,14)}$.
The most appropriate empirical antibiotic therapy must be selected based on the individual clinical case and take into consideration the epidemiological scenario present in each institution. An early approach is required in cases of sepsis/septic shock. In addition, a broad-spectrum antimicrobial therapy must be initiated and adjusted as soon as antimicrobial susceptibility testing results become available since increased chance of clinical success are associated with early adjustment of antimicrobial therapy ${ }^{(14)}$.

Currently, after bloodculture positivity, the laboratory requires on average 1 hour to conduct bacterioscopy and release a partial result (Gram positive bacteria, Gram negative or yeast) and at least 18 hours for bacterial colony isolation. Final results require on average 24 to 48 additional hours for completing identification and antimicrobial susceptibility tests. Additionally, only after this period the laboratory report is released and the antimicrobial therapy is adjusted ${ }^{(9)}$.

Therefore, new technologies are vital in order to reduce the time for bacterial identification. The advances in molecular biology methods have enabled the reduction in identification time and polymerase chain reaction (PCR) is one of the most

First submission on 22/12/12; last submission on 22/10/12; accepted for publication on 12/12/12; published on 20/06/13

1. Master degree in Infectious Diseases by Universidade Federal de São Paulo (UNIFESP).

2. PhD in Infectious Diseases by UNIFESP; attending post-doctorate at UNIFESP and laboratory supervisor at ALERTA laboratory from UNIFESP Infectious Disease Department.

3. Infectious Disease doctor; attending Master's in Infectious Diseases at UNIFESP.

4. PhD in Medicine by UNIFESP; sandwich PHD at University of Iowa Pathology Department; associate professor and ALERTA laboratory director from UNIFESP Infectious Disease Department; researcher level 1A at the National Council for Scientific and Technological Development (Conselho Nacional de Desenvolvimento Científico e Tecnológico [CNPq]); coordinator at the Bacteriology Committee of the Brazilian Society of Infectious Diseases (Comitê de Bacteriologia da Sociedade Brasileira de Infectologia [SBI]). 
sensitive methods, which may be applied for the identification of slow growing and non-cultured microorganisms. Nevertheless, several identification tests based on PCR use the amplification of conserved genes such as those from the portion $16 \mathrm{~S}$ of ribosomal deoxyribonucleic acid (rDNA). This technique normally requires the extraction of nucleic acids from bacterial colony isolates, which demands on average 24 to 36 hours. PCR conducted directly from clinical samples is usually restricted to specific samples such as spinal fluid, while as reaction inhibition may occur with some samples. Mass spectrometry has been commonly used by research laboratories and it is regarded as a promising technique as to the rapid identification of microorganisms (Figure 1).

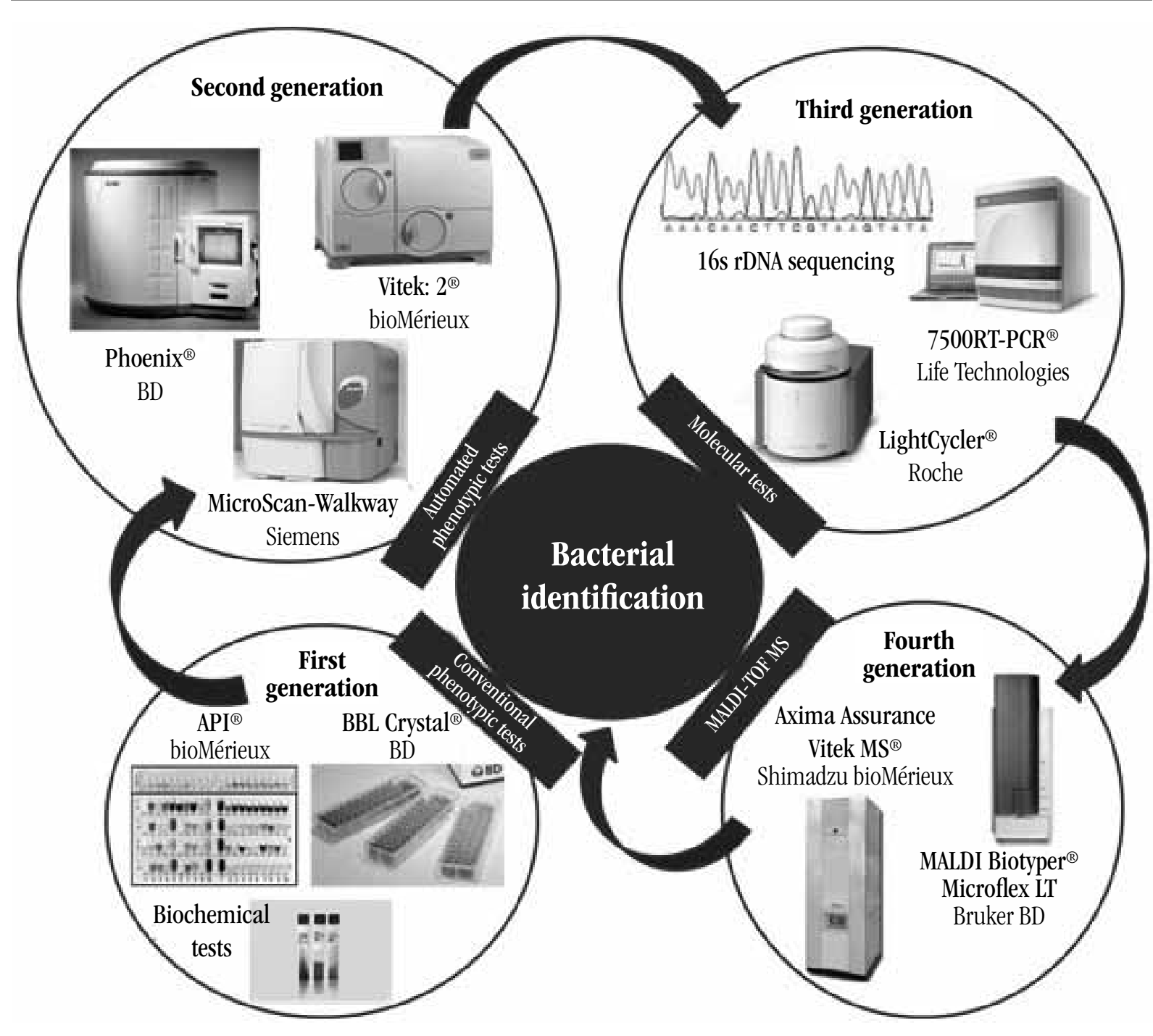

FIGURE 1 - Evolution of bacterial identification in the microbiology laboratory

rDNA: ribosomal deoxyribonucleic acid; MALDI-TOF MS: matrix assisted laser desorption/ionization-time of flight mass spectrometry.

\section{Mass spectrometry applied to the identification of human pathogens}

Mass spectrometry (MS) is another method that may be of great help to microbiological diagnosis. Furthermore, it has been widely used as a research tool, mainly in proteomic and lipid analyses. Several techniques based on ionization and subsequent biomolecular detection have been developed and the matrix assisted laser desorption/ionization-time of flight (MALDI-TOF) has been one of the mostly applied. It is a "mild" ionization technique, in which the formed ions have low internal energy, allowing the observation of ionized molecules with little or no fragmentation. Ion production from high molecular mass and non-volatile compounds such as 
proteins has expanded the use of MS to several forms of molecules and study fields, mainly in the clinical microbiology laboratory. It is a rapid method for bacterial and fungal identification, with high accuracy and low operating costs ${ }^{(23,26)}$.

Fresh colonies $(<24 \mathrm{~h})$ are preferentially selected for performing bacterial identification by MALDI-TOF MS. However, it also can be accomplished using older colonies stored in air ambient. The identification may be conducted directly from the bacterial colonies or after total protein extraction. In the former one, a microorganism colony is inoculated in the plate for mass spectrometry. In the latter, $1 \mu \mathrm{l}$ of the extract is inoculated in the plate. There are several extraction protocols, either prescribed by MALDI-TOF MS manufacturers or published in the literature, which are specific for certain groups of microorganisms such as fungi and mycobacterium ${ }^{(12)}$. Subsequently, $1 \mu \mathrm{l}$ of the organic matrix, usually cinnamic acid, is added to the sample. Afterwards, the plate is positioned in the equipment for MALDI-TOF MS analysis (Figure 2). In all cases, it is recommended that the sample analysis should be carried out in duplicate.

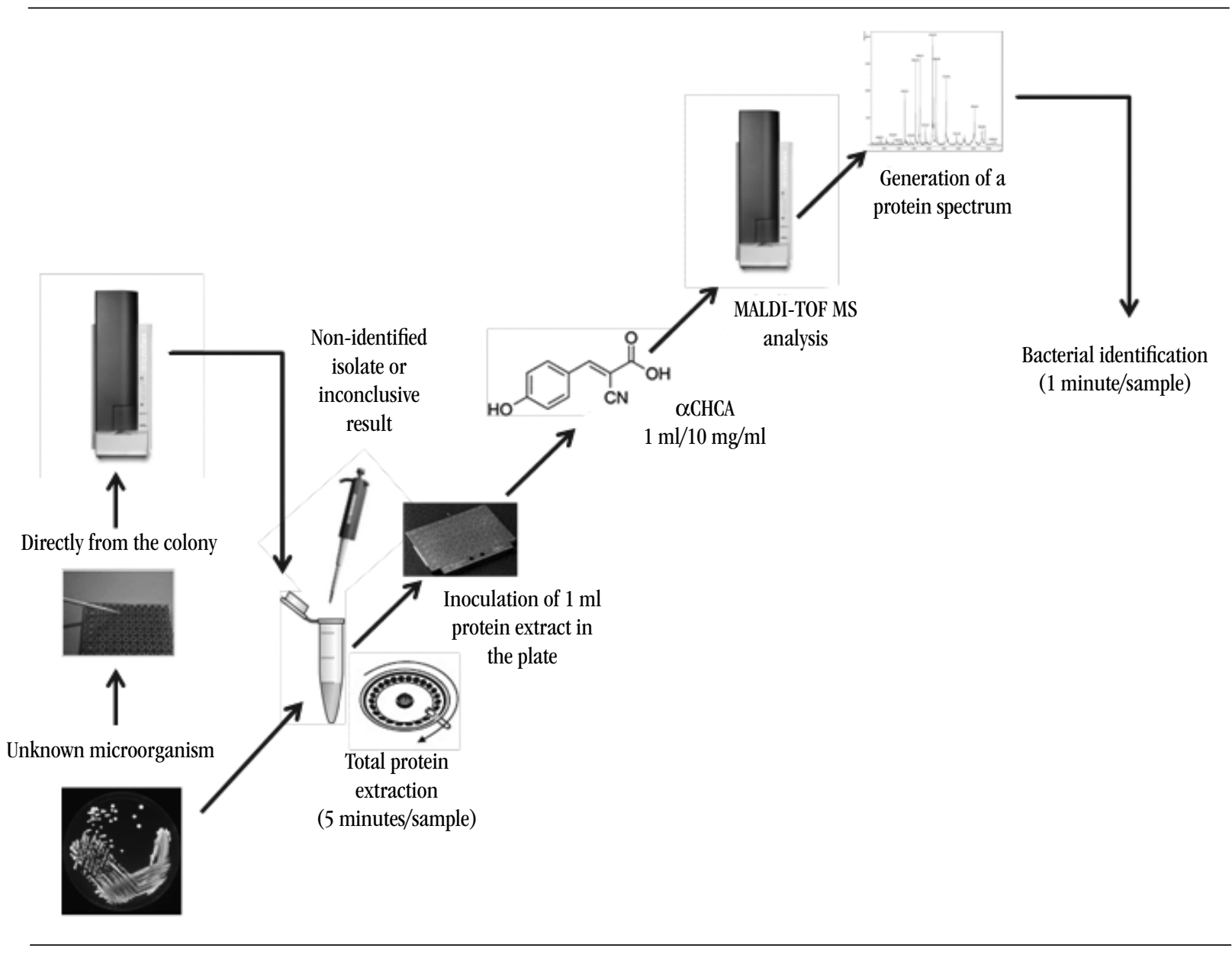

FIGURE 2 - Flowchart of the use of MALDI-TOF MS in microorganism identification at the clinical microbiology laboratory

MALDI-TOF MS: matrix assisted laser desorption/ionization-time of flight mass spectrometry; $\alpha$ CHCA: alpha-cyano-4-hydroxycinnamic acid.

The matrix allows that the proteins, non-volatile and thermally labile compounds, may be ionized and form intact ions in the gaseous phase. A laser beam, infrared or ultraviolet pulsed, serves as an ionization or desorption source. The matrix absorbs the laser energy, inducing the partial vaporization of the sample. As soon as the vaporization and ionization of molecules occur, they are transferred electrostatically to a mass spectrometer vacuum chamber. The ionized particles are formed and the migration of these particles is proportional to the ratio charge:mass, that is to say that minor ions reach a TOF detector before larger ions. The identification by MALDI-TOF MS is based on the score value released by the equipment. A higher or similar 2.3 value indicates that the identifications of genus and species are reliable. 2.0 to 2.29 show that the genus is reliable and the species is probable. 1.7 
to 1.99 values indicate that the identification of genus is probable. When score values are lower than 1.7, the equipment result is released as "not reliable", evincing that spectra acquisition was insufficient or no peak protein was detected, and further analyses are required for this sample. The optimal reproducibility in microbial identification by MALDI-TOF MS is based on the assessment of ribosomal proteins, which are commonly abundant in the cell. These proteins are part of the cell translational apparatus and are present in all living cells. Therefore, MALDITOF MS is minimally influenced by environmental conditions or microorganism growth ${ }^{23,26,29)}$.

Currently, in Brazil, MS systems used for bacterial identification are available from two companies: the German Bruker Daltonics (Bremen, Germany) and the Japanese Shimadzu (Kyoto, Japan). Bruker mass spectrometer, Microflex LT (Linear TOF), is represented by BD Diagnostics (Becton, Dickinson and Company-Franklin Lakes, EUA), whereas Axima-Assurance from Shimadzu is commercialized by bioMérieux (Marcy lEtoile, France) as Vitek MS. The pieces of equipment from both manufacturers present the same principles, with relative differentiation in the number of spectra stored in their respective databases. Nonetheless, a study comparing both systems demonstrated that Bruker Daltonics mass spectrometer yields better results as to Gram positive bacteria in comparison with Shimadzu, whereas there was no significant difference as to Gram negative bacteria ${ }^{(5)}$. In other two studies, similar results were observed for the identification of Gram positive and Gram negative bacteria when both systems were compared ${ }^{(6,22)}$.

One of the most significant points a to the success of this technique in microbiology laboratories is precisely the mass spectrometry database, which allows data interpretation. It is a fingerprinting of the microorganism in test. The main databases available for the identification of microorganisms are MALDI Biotyper - Bruker Daltonics (concept: Main Spectrum analyses) and AnagnosTec SARAMIS (Spectral ARchive And Microbial Identifications System) - bioMérieux (concept: Super Spectrum and Advanced Spectra Classifier [ASC]).

The Biotyper system was conceived and marketed exclusively by Bruker Daltonics and is capable of analyzing specimens including bacterial, mycobacterial, and fungal samples in addition to samples recovered directly from positive blood culture bottles. The SARAMIS database was previously created and maintained by AnagnosTec $\mathrm{GmbH}$ prior to being purchased by bioMérieux for incorporation into the Vitek-MS platform. There is another database called Andromas system, utilized predominantly for clinical diagnostics in Europe and manufactured and maintained by Andromas SAS (Paris, France). Some infrequent bacterial genera or environmental bacteria could not be identified by MALDI-TOF MS since their protein profiles are not included in the database. When the sample spectra is acquired by the system but not recognized by the database, it is possible to include its spectra into the individual database (library), but the operator must be responsible for providing or adding accurate information or data to the system. Thus, the inserted data should include reference strains or those whose identification has been confirmed by molecular biology techniques. MALDI Biotyper software allows the creation of a spectrum database different from that provided by the system. MALDITOF MS databases may also be improved with updates offered by the manufacturers ${ }^{(12,25)}$.

In an investigation carried out by El Khéchine $e t a l .^{(12)}$, the authors created a local database, complementary to MALDI Biotyper 2.0 (Bruker Daltonics), with the most frequent mycobacterial species isolated in recent years within that geographic region. This database was established from previously stored samples: 11 mycobacterial strains including seven species from M. tuberculosis complex, 12 mycobacterial strains including 11 species from M. avium complex and 20 mycobacterial strains including 16 species from non-tuberculosis mycobacteria (NTM). An original MALDI-TOF MS identification protocol was used for inactive mycobacteria. By applying this protocol in a standardized inoculum (at least $10^{5} \mathrm{UFC} / \mathrm{ml}$ ) and linking the databases, it was possible to identify 87 M. tuberculosis, $25 \mathrm{M}$. avium and 12 NTM isolates, with identification score $\geq 2$ within merely 2.5 hours $^{(12)}$.

Although the inclusion of new protein spectra into MALDITOF MS databases is an alternative to circumvent the problem of non-identified microorganisms, the recently developed software releases that probably will be available at clinical microbiology laboratories do not allow this insertion due to the new regulations introduced by the inspecting agencies. Therefore, periodic database updates provided by the manufactures are crucial for a wider coverage of the surveyed microorganisms.

Several studies that evaluated the performance of MALDITOF MS in microorganism identification demonstrated that these systems are highly descriptive, accurate and reproducible. In a retrospective investigation carried out by Eigner et al. ${ }^{(11)}, 1,116$ clinical isolates were analyzed comparing Microflex LT (software MALDI-Biotyper 2.0 - Bruker Daltonics) with conventional biochemical identification. The study demonstrated a 95.2\% consistency in the identification of bacterial species ${ }^{(11)}$. In another research, Cherkaoui et al.$^{\left({ }^{6}\right)}$ compared MALDI-TOF MS Microflex LT and Axima Assurance with automated and/or phenotypic methods. The sequencing of portion 16s rDNA was used as gold standard in order to clarify discrepant results among the methods. The authors analyzed 720 clinical isolates consecutively collected during laboratory routine. Microflex LT equipment identified 680 isolates (94.4\%), from which 674 were 
consistent with molecular and phenotypic tests, whereas Axima Assurance system identified 639 isolates (88.8\%), from which 635 were also consistent. The study concluded that if MALDITOF MS had been applied as initial test and the biochemical identification had only been applied when there was no identification, the laboratory would have saved approximately US $\$ 5,00$ per isolate and the time for result release would have been reduced to approximately 8 hours ${ }^{(6)}$.

Investigations that made use of more recent databases reported approximately $100 \%$ identification of some microorganisms such as Neisseria spp. ${ }^{(18)}$, Clostridium spp. ${ }^{(16)}$, Mycobacterium spp. ${ }^{(25)}$ and Salmonella spp. ${ }^{(10)}$. Furthermore, MALDI-TOF MS method has been used in yeast identification ${ }^{(21)}$. Marklein et al. ${ }^{(21)}$ tested 250 clinical isolates of Candida spp. from different body sites and $96 \%$ of the samples were accurately identified. Although the yeast results are promising, technical advances are still required for filamentous fungi such as Aspergillus spp., Penicillium spp. and Fusarium spp.

\section{Bacterial and yeast identification directly from clinical samples}

The possibility of identifying pathogens directly from clinical samples such as urine or positive bloodculture has aroused great interest ${ }^{(20)}$. The high concentration of proteins present in the blood and urine interferes in the detection of specific bacterial and fungal proteins, hence a major hindrance in the use of this method. Besides, the low inoculum of microorganisms present in the samples also hampers the identification of mass spectra. Thus, several research groups have developed and assessed different protocols for the extraction of proteins from microorganisms and removal of substances that may interfere in MALDI-TOF MS analysis ${ }^{(2,13,20,28)}$. These methods may be laborious and include several phases such as centrifugation, wash, gel separation and protein extraction.

Studies on bacterial identification by MALDI-TOF MS, directly from positive bloodculture, have yielded better results in the identification of Gram negative organisms in comparison with Gram positive. Ferreira et al. . $^{(3)}$ obtained an accurate bacterial identification of $83.3 \%$ Gram negative bacteria and $31.8 \%$ Gram positive bacteria ${ }^{(28)}$ in comparison with conventional methods. In contrast, Moussaoui et $a l .{ }^{(24)}$ yielded better results with the accurate identification of $91.1 \%$ Gram negative and $89 \%$ Gram positive microorganisms through MALDI-TOF MS.

Buchan et al. ${ }^{(2)}$ evaluated 164 positive bloodculture samples with Sepsityper kit (Bruker Daltonics) for bacterial extraction. According to these authors, MALDI Sepsityper kit was able to identify $85.5 \%$ of isolates directly from monomicrobian bloodculture samples, with $97.6 \%$ genus consistency and 94.1\% species consistency in comparison with other routine identification methods. Analyzing Gram negative microorganisms, 97.6\% of tested samples reached a reliable confidence score (2.0). Analyzing Gram positive isolates, $80 \%$ presented acceptable score. However, when the analysis parameters of specific spectra for bloodculture were altered, there was an improvement in the identification of Gram positive samples ( $80 \%$ to $89 \%$ ). The average identification time for MALDI-TOF biotyper/sepityper was 23 to 83 hours faster than other routine methods for the identification of Gram positive isolates and 34 to 51 hours for Gram negative isolates ${ }^{(2)}$. Vlek et al. ${ }^{(29)}$ demonstrated that bacterial identification by MALDI-TOF MS, directly from bloodculture, reduced the identification time in 28.8 hours and it was associated with an increase of $11.3 \%$ in the number of patients that underwent suitable antimicrobial therapy 34 hours after bloodculture positivity.

As to urine samples, Ferreira et al. ${ }^{(13)}$ revealed a $93.1 \%$ consistency between MALDI-TOF MS identification and conventional methods. It is particularly worth mentioning that, although microorganism identification by MALDI-TOF MS directly from clinical samples is a promising method, the protocols still need to be improved. Moreover, the antimicrobial susceptibility tests are still required by conventional methods.

\section{MALDI-TOF MS applied to microbiological diagnostics: advantages and disadvantages}

As it was discussed previously, the identification by MALDI-TOF MS is based on the analysis of protein spectrum from bacterial ribosome, therefore it is closely related to the analysis of gene $16 \mathrm{~S}$ rDNA sequence. Nevertheless, due to the great similarity of these sequences in some species such as Shigella spp. and Escherichia coli or Streptococcus pneumoniae and other members of $S$. oralis/mitis group, sometimes there may be divergence in the identification among these species. Accordingly, standard biochemical tests such as antigen detection or molecular methods are required ${ }^{(30)}$.

Other applications of MALDI-TOF MS are in development and the possibility of detecting bacterial resistance has aroused great interest. Several studies have demonstrated the detection of $\beta$-lactamase production in enterobacteria, the main mechanism of resistance to carbapenems and other $\beta$-lactam antibiotics ${ }^{(4,17)}$. The detection principle is based on the hydrolysis of $\beta$-lactam ring in presence of bacterial enzymes. MALDI-TOF MS technique is able to detect antibiotic mass alteration due to the chemical modification in the antimicrobial molecule. For this test, it 
is necessary to incubate the bacterial protein extract with the antibiotic for a period of $1-4$ hours so that it is possible to detect the presence of $\beta$-lactamase with hydrolytic activity on penicillins, cephalosporins and/or carbapenems ${ }^{(4,27)}$.

\section{CONCLUSION}

Within the past years, clinical microbiology laboratories experienced revolutionary changes in the way in which microorganisms are identified.

The major advantage of MALDI-TOF MS is the reliability as to the identification through the analysis of bacterial protein profile instead of physical, biochemical and metabolic differentiation. In comparison with conventional phenotypic methods or PCR, MALDI-TOF MS has been increasingly applied in the microbiology diagnostics and it has started to replace conventional biochemical methods in some laboratories worldwide. In Brazil, it is being tested by research labs and recently, Shimadzu system was approved for in vitro diagnostic (IVD) use, while Bruker system is waiting for ANVISA approval, scheduled for the second half of 2013. In Europe, this technique has been used in microbiology clinical laboratories since 2009. MALDI-TOF MS has been recently introduced in diagnostic microbiology and several innovations and developments indicate its promising use in the future.

\section{TRANSPARENCY DECLARATION}

AFS works at Becton Dickinson. ACG has received supplies from bioMérieux for conducting scientific research. The other authors have no conflict of interests.

\section{RESUMO}

A rápida identificação de microrganismos no laboratório de microbiologia clínica é de extrema importância para direcionar o manejo e o tratamento de pacientes. Geralmente, a identificação bacteriana por métodos bioquímicos convencionais necessita de 18 a 24 horas para o crescimento e o isolamento da colônia bacteriana e, pelo menos, 24 boras adicionais para a identificação da espécie. Novas tecnologias em microbiologia têm focado no desenvolvimento de métodos relacionados com o diagnóstico rápido das infecções da corrente sanguínea, uma vez que essas infecções são associadas à alta morbimortalidade.

Unitermos: MALDI-TOF MS; espectrometria de massa; infecção da corrente sanguínea; infecções bacterianas; diagnóstico rápido.

\section{REFERENCES}

1. ANGUS, D. C. et al. Epidemiology of severe sepsis in the United States: analysis of incidence, outcome, and associated costs of care. Crit Care Med, v. 29, n. 4, p. 1303-10, 2001.

2. BUCHAN, B.; RIEBE, K.; LEDEBOER, A. Blood culture bottles identification of bacteria from positive routine microbiological methods for using sepsityper specimen processing to comparison of the MALDI biotyper system.J Clin Microbiol, v. 50, n. 2, p. 346-52, 2012.

3. BURCHARDI, H.; SCHNEIDER, H. Economic aspects of severe sepsis: a review of intensive care unit costs, cost of illness and cost effectiveness of therapy. Pharmacoeconomics, v. 22, n. 12, p. 793-813, 2004.

4. BURCKHARDT, I.; ZIMMERMANN, S. Using matrix-assisted laser desorption ionization-time of flight mass spectrometry to detect carbapenem resistance within 1 to 2.5 hours. J Clin Microbiol, v. 49, n. 9 , p. 3321-4, 2011.
5. CARBONNELLE, E. et al. Robustness of two MALDI-TOF mass spectrometry systems for bacterial identification. J Microbiol Methods, v. 89, n. 2, p.133-6, 2012.

6. CHERKAOUI, A. et al. Comparison of two matrix-assisted laser desorption ionization-time of flight mass spectrometry methods with conventional phenotypic identification for routine identification of bacteria to the species level. J Clin Microbiol, v. 48, n. 4, p. 1169-75, 2010.

7. CRIBBS, S. K.; MARTIN, G. S. Expanding the global epidemiology of sepsis. Crit Care Med, v. 35, n. 11, p. 2646-8, 2007.

8. DANAI, P; MARTIN, G. S. Epidemiology of sepsis: recent advances. Curr Infect Dis Rep, v. 7, n. 5, p. 329-34, 2005.

9. DELLINGER, R. P. et al. Surviving sepsis campaign: international guidelines for management of severe sepsis and septic shock: 2008. Crit Care Med, v. 36, n. 1, p. 296-327, 2008.

10. DIECKMANN, R. et al. Rapid classification and identification of Salmonellae at the species and subspecies levels by whole-cell matrix- 
assisted laser desorption ionization time of flight mass spectrometry. Appl Environ Microbiol, v. 74, n. 24, p. 7767-78, 2008.

11. EIGNER, U. et al. Performance of a matrix-assisted laser desorption ionization-time-of-flight mass spectrometry system for the identification of bacterial isolates in the clinical routine laboratory. Clin Lab, v. 55, n. 7-8, p. 289-96, 2009.

12. EL KHÉCHINE, A. et al. Matrix-assisted laser desorption/ionization time-of-flight mass spectrometry identification of mycobacteria in routine clinical practice. PLOS ONE, v. 6, n. 9, p. e24720, 2011.

13. FERREIRA, L. et al. Microorganisms direct identification from blood culture by matrixassisted laser desorption/ionization time-of-flight mass spectrometry. Clin Microbiol Infect, v. 17, n. 4, p. 546-51, 2011.

14. GARNACHO-MONTERO, J. et al. Impact of adequate empirical antibiotic therapy on the outcome of patients admitted to the intensive care unit with sepsis. Crit Care Med, v. 31, n. 12, p. 274251, 2003.

15. GREUB, G.; PROD'HOM, G. Automation in clinical bacteriology: what system to choose? Clin Microbiol Infect, v. 17, n. 5, p. 655-60, 2011.

16. GROSSE-HERRENTHEY,A. et al. Challenging the problem of clostridial identification with matrix-assisted laser desorption and ionization-timeof-flight mass spectrometry (MALDI-TOF MS). Anaerobe, v. 14, n. 4, p. 242-9, 2008.

17. HRABÁK, J. et al. Carbapenemase activity detection by matrix-assisted laser desorption ionization-time of flight mass spectrometry. J Clin Microbiol, v. 49, n. 9, p. 3222-7, 2011.

18. ILINA, E. N. et al. Direct bacterial profiling by matrix-assisted laser desorption-ionization time-of-flight mass spectrometry for identification of pathogenic Neisseria.J Mol Diagn, v. 11, n. 1, p. 75-86, 2009.

19. KUMAR, A. et al. Duration of hypotension before initiation of effective antimicrobial therapy is the critical determinant of survival in human septic shock. Crit Care Med, v. 34, n. 6, p. 1589-96, 2006.

20. LA SCOLA, B. Intact cell MALDI-TOF mass spectrometry-based approaches for the diagnosis of bloodstream infections. Expert Rev Mol Diagn, v. 11, n. 3, p. 287-98, 2011.
21. MARKLEIN, G. et al. Matrix-assisted laser desorption ionization-time of flight mass spectrometry for fast and reliable identification of clinical yeast isolates. J Clin Microbiol, v. 47, n. 9, p. 2912-7, 2009.

22. MARTINY, D. et al. Comparison of the microflex LT and Vitek MS systems for routine identification of bacteria by matrix-assisted laser desorption ionization-time of flight mass spectrometry. J Clin Microbiol, v. 50, n. 4, p. 1313-25, 2012.

23. MARVIN, L. F; ROBERTS, M. A.; FAYA, L. B. Matrix-assisted laser desorption/ionization time-of-flight mass spectrometry in clinical chemistry. Clin Chim Acta, v. 337, n. 1-2, p. 11-21, 2003.

24. MOUSSAOUI, W. et al. Matrix-assisted laser desorption ionization time-of-flight mass spectrometry identifies $90 \%$ of bacteria directly from blood culture vial. Clin Microbiol Infect, v. 16, n. 11, p. 1631-8, 2010.

25. PIGNONE, M. et al. Identification of mycobacteria by matrix-assisted laser desorption ionization-time-of-flight mass spectrometry. J Clin Microbiol, v. 44, n. 6, p. 1963-70, 2006.

26. SENG, P. et al. Ongoing revolution in bacteriology: routine identification of bacteria by matrix-assisted laser desorption ionization time-of-flight mass spectrometry. Clin Infect Dis, v. 49, n. 4, p. 543-51, 2009 .

27. SPARBIER, K. et al. Matrix-assisted laser desorption ionization-time of flight mass spectrometry-based functional assay for rapid detection of resistance against $\beta$-lactam antibiotics. J Clin Microbiol, v. 50, n. 3 , p. 927-37, 2012.

28. STEVENSON, L. G.; DRAKE, S. K.; MURRAY, P. R. Rapid identification of bacteria in positive blood culture broths by matrix-assisted laser desorption ionization-time of flight mass spectrometry. J Clin Microbiol, v. 48, n. 2, p. 444-7, 2010.

29. VLEK, A. L. M. et al. Direct matrix-assisted laser desorption ionization time of-flight mass spectrometry improves appropriateness of antibiotic treatment of bacteremia. PLOS ONE, v. 7, n. 3, p. e32589.

30. WIESER, A. et al. MALDI-TOF MS in microbiological diagnosticsidentification of microorganisms and beyond (mini review). Appl Microbiol Biotechnol, v. 93, n. 3, p. 965-74, 2012.

\section{MAILING ADDRESS}

\section{Anderson Fernandes Santos}

Laboratório ALERTA; Universidade Federal de São Paulo; Rua Pedro de Toledo, 781, $0^{\circ}$ andar, fundos; Vila Clementino; CEP: 04039-032; São Paulo-SP, Brazil; e-mail: fernandes.anderson@gmail.com. 